\title{
A review of the verbal memory profile of individuals with autism spectrum disorder
}

\begin{abstract}
Behavioral studies concerning the memory of individuals with Autism Spectrum Disorder (ASD) have yielded inconsistent results over the past few decades. Some have found impairment where as others have reported an intact memory profile in ASD. Therefore, in the present review, we will examine the verbal memory profile of ASD. A systematic search has located 18 articles for review and analysis. It is suggested that ASDs mainly have an intact verbal recognition performance but impaired verbal recall ability. The conflicting memory performance found in ASD may probably be due to confounding factors, including differences in the experimental designs of research studies, the intellectual functioning levels of ASD and their developmental trajectories. In general, the memory processing of ASD is implicated by the frontal lobe function, and the memory problem is primarily caused by the poor utilization of organizational strategies during information encoding. A failure to encode the information properly will directly hinder their later retrieval performance. Finally, priorities for future research in the memory functions of ASD are suggested, and the need for more rigorous research paradigms is highlighted. With a more comprehensive understanding of the underlying mechanism of the memory functions of those with ASD, it is believed that better intervention programs can be developed to aid their memory deficits and to improve their daily-life performances.
\end{abstract}

Keywords: autism spectrum disorder, verbal memory, recall and recognition, task complexity, chronological age, functioning level
Volume 2 Issue I - 2015

\author{
Nicolson Yat Fan Siu,' Jacqueline Jiaying LE' \\ 'Department of Psychology, The Chinese University of Hong \\ Kong, China \\ 2Liberal Studies Department,YMCA of Hong Kong Christian \\ College, China
}

\section{Correspondence: Nicolson, Yat-Fan SIU, Department of Psychology, The Chinese University of Hong Kong, Shatin, Hong} Kong, Tel 852 3946I26I, Email yfsiu@psy.cuhk.edu.hk

Received: November 21, 2014 | Published: December 23, 2014

\section{Introduction}

ASD is aneuro-developmental disordercharacterized by disturbance in communication and social interaction, restricted interests, and stereotyped behaviors. ${ }^{1}$ Previous findings have attributed the cause of ASD both to genetic contribution and environmental influences. ${ }^{2,3}$ Yet, with the advancement in neuro-imaging techniques, there is now an even greater insight into the neurobiology perspective and more evidence of the involvement of the neuro-developmental component in ASD. ${ }^{4,5}$ The prevalence of ASD has been increasing during the past decades. A study has found that the number of diagnosed cases of ASD in the 2000s is double that of the prevalence rate as reported in the1980s. ${ }^{6}$ Apart from the genuine rise in the number of children with ASD, greater awareness and changes in the diagnostic criteria have contributed to an increase in the cases reported. ${ }^{7,8}$ The current estimation of the rate of individuals with ASD has increased to 110 per $10,000 .{ }^{9}$

\section{Autism Spectrum Disorder as a Frontal Disorder}

Higher cortical functions have been reported to be impaired in the ASD population, yet their severity has varied. Whereas some individuals with ASD show severe impairments in general, others show isolated cognitive dysfunctions. ${ }^{10,11}$ In recent years, three key common theories have been explored in an attempt to explain the brains and the behaviors of those with ASD. First, is the theory of mind deficit. ${ }^{12}$ Neuro-physiological studies and neuro-imaging have confirmed that children with ASD develop their theory of mind much more slowly than normal developing children and show less activation of the brain regions corresponding to their theory of mind. ${ }^{13-16}$ Second, is the theory of weak central coherence? This theory suggests that individuals with ASD have a special information processing style rather than a deficit. ${ }^{10,17,18}$ However, further investigations of the brain basis of central coherence are required to confirm this idea.
Although these two theories together have explained many deficits and characteristics of ASD, the executive dysfunction theory might be the best one to account for ASD on its own. It has been suggested that ASD is a frontal disorder of the executive dysfunctions. ${ }^{19}$ There are similarities in cognitive and behavioral presentations between individuals with ASD and individuals with frontal lesions. The abnormalities found in individuals with ASD, such as the lack of motivation to communicate, poor social interaction, and repetitive behaviors, are suggested to be related to the atypical functioning of the frontal lobe $\mathrm{e}^{20-22}$ for review.

\section{Memory and Frontal Lobe}

The frontal lobe is located in the anterior part of each cerebral hemisphere of the brain. It is the largest structure in the brain and it constitutes about one-third of the whole brain volume. The frontal lobe is thought to play a central role in the executive control of the higher cortical functions such as memory, planning, and organization capability. ${ }^{23-25}$ In 1935, it was first suggested that the frontal cortex was involved in memory. A study on frontal-lobe-lesioned monkeys found that they perform well on immediate response but that they fail when delayed ${ }^{26}$ and for human beings with frontal injuries, they perform normally on classic memory tests. ${ }^{27-28}$ but on examination with extend neuropsychological memory assessments, the results show that there is a close relation between the frontal lobe and memory. ${ }^{29-31,25,26}$

It has been suggested that the frontal cortex plays a central role in organizing memory. ${ }^{32-34}$ This is crucial in facilitating memory encoding and retrieval, especially when there is a large amount of information to remember. The behavioral data of patients with frontal lobe dysfunctions or lesions have further implicated the role of the frontal lobe in selecting and initialing an organizational strategy to enhance the encoding of new information and retrieval. ${ }^{35-38}$ In addition, in Positron Emission Tomography (PET) studies, the encoding and 
retrieval of verbal information have led to prefrontal activation in which encoding has been more dependent on the left prefrontal cortex. ${ }^{39,40}$ The regional cerebral blood flow in the frontal regions was found to be correlated with the rate of encoding. ${ }^{41}$ The faster the rate of encoding, the larger the amount of blood flows to the frontal regions when it consumes more energy. One study has also suggested that when the encoding materials require self-organization, the activation of the dorsal-lateral prefrontal cortex would even be greater. ${ }^{42}$ In other words, when the materials are presented in a random order, the effort to rearrange them into categories based on their semantic relations requires the involvement of the prefrontal cortex. When individuals lose the ability to self-organize the presented materials, it may result in poor memory performance. Some studies have found that memory encoding deficits would lead to retrieval deficits. ${ }^{43,44}$ If the information is not learnt in an effective way, there will be greater interference and memory loss during the process.

\section{Role of Frontal Lobe Function Implicated in Memory Processing of ASD}

There is increasing evidence of the involvement of the frontal lobe in the memory processing of ASD. The results of neuropsychological testing on individuals with ASD are suggestive with regard to the frontal-lobe-related memory deficits. Individuals with ASD are reported to adopt less effective strategies to facilitate memory encoding and retrieval than normal developing individuals. There is evidence to support that ASD individuals have reduced neural connectivity and information processing deficits when utilizing cognitive strategies for the efficient encoding of information. ${ }^{45}$ With a failure to initiate organizing strategies, ASD individuals would learn new materials inefficiently and show a greater impairment when the complexity of the materials increases. ${ }^{46,47}$ They cannot use category information to aid their recall. ${ }^{48}$ Various studies have also shown that ASD individuals utilize a different organization and processing of memory from normal developing individuals. ${ }^{49,50}$ These memory performances found in individuals with ASD are parallel to individuals with dysfunctional or damaged frontal lobes.

\section{Memory in Autism}

Memories being commonly known as the ability to store retain and recall information and experience, is related to everything in our lives. We are constantly making decisions and basing our actions on them. Over the past few decades, the memory performance of ASD individuals has been widely investigated. However, the results are inconsistent across different studies. Verbal memory is a broad term which commonly refers to the memory of language in its various forms. It is typically assessed by neuropsychological tests that measure the memory of a list of words, sentences or even a comprehensive story. To mention a few, the California Verbal Learning Test, ${ }^{51}$ the Hong Kong List Learning Test (HKLLT), ${ }^{52,53}$ the Rey Auditory Verbal Learning Test ${ }^{54,55}$ and the Verbal Intelligence Scale (VIQ) from the Wechsler Adult Intelligence Scale - Revised (WAIS) $-\mathrm{R}^{56,57}$ are widely used verbal memory tests. There have been numerous studies which investigate the verbal memory functions of individuals with autism over the past years, but the results have been inconclusive. It may therefore be beneficial to review the previous articles to enhance our understanding of verbal memory functions in ASD individuals. With a closer examination of each of the studies, it may be possible to ascertain the reasons which lead to the different results and hence provide more clues for further studies. Interventions and special services may develop accordingly to assist them to perform better in their daily lives.

\section{Method}

The articles for examination were identified through a comprehensive literature search from various academic search engines, namely, PsycINFO, MEDLINE, and Embase. Six-hundredand-twenty-seven articles resulted from this search 174 from PsycINFO;277 from MEDLINE; and 176 from Embase. These articles were identified by key words searches by combining terms of verbal memory, verbal recall, verbal learning, verbal recognition, autism, autistic, and Asperger syndrome. Studies on individuals with comorbid ASDs and other developmental disorders were excluded from the analysis. Only empirical studies with measures of verbal memory performance of ASD were selected. In addition, studies are required to include age and verbal ability matched control as reference to ensure a fair comparison with the verbal-performance ASD. Finally, 19 studies were chosen and included for analysis (Figure 1).

\section{Discussion}

\section{Performance on Verbal Recognition}

The performance on verbal recognition is considered to be more consistent. Most of the reviewed studies have reported that verbal recognition of ASD is intact. ${ }^{58-61}$ When ASD individuals were provided with a new list of words to recognize and then asked whether the items have been previously presented or not, their performance on correct identification was comparable with normal developing individuals. ASD individuals were able to achieve a performance comparable to that of normal developing individuals even in a delayed recognition condition. ${ }^{45,58,60}$ It is suggested that ASD individuals were able to consolidate and store the information which is mediated by the temporal lobes. ${ }^{62}$ In addition, there also is evidence which shows that ASD individuals commit similar levels of conservative response bias when compared with normal developing individuals in verbal recognition tasks. This indicates that both normal developing individuals and ASDs have a tendency to favor "no" responses and this suggests that both groups are using similar decision criteria on verbal recognition. In other words, they use similar verbal recognition strategies. ${ }^{60}$ However, ASDs have also been reported to commit more false alarm errors, and this indicates that they are more susceptible to interference from irrelevant information. ${ }^{63}$

\section{Performance on Verbal Recall}

Regarding the performance of ASDs on verbal recall, previous literatures have reported inconsistent results. Nevertheless, it is believed that there are some degrees of impairments in general. A number of reviewed studies have reported that ASD individuals were impaired in verbal recall tasks. ${ }^{45-46,48,58-60,63-65}$ ASDs showed poorer performance on free recall, and the severity of impairment increased from single word recall to sentence recall and then to story recall. However, there were no differences between the performance of immediate free recall and long-delayed recall. ${ }^{45,61}$ It is suggested that individuals with ASD were able to maintain the information stored once they have encoded it accordingly. On the other hand, individuals with ASD, but not the normal developing individuals, have shown improvement from free to cued recall. When they were provided with external support during the retrieval of information, they were able to achieve better performances. ${ }^{58,61}$ It gives concrete evidence to support the Task Support Hypothesis (TSH) ${ }^{65}$

\section{Problems of Encoding and Retrieval}

Verbal memory deficits in ASDs may be caused by the impairments in encoding and retrieval of information this is asthe majority of 
studies have suggested. They have given evidence to support that there is reduced neural connectivity and information processing deficits in utilizing cognitive strategies for the efficient encoding of information in autistic individuals. ${ }^{45}$ With a failure to initiate proper organizing strategies, ASD would not be able to learn new materials efficiently and hence show a greater impairment when the complexity of materials increased. ${ }^{46,47}$ It seems that ASDs fail to categorize the information to aid their recall. ${ }^{48}$ Studies have also shown that ASDs utilize an organization and processing of memory which is different from normal developing individuals. ${ }^{49,50}$ Therefore, more studies will have to be done to achieve a deeper understanding of its underlying mechanism.

In addition to encoding problems, ASDs suffer from difficulty in retrieving information. Several studies have shown that ASDs who were impaired in the free recall of words or stories were able to perform as well as normal developing individuals when given cues or recognition choices. ${ }^{45,58}$ This provided concrete evidence that ASD individuals have difficulties in retrieving information. Research with a more vigorous paradigm design will provide more concrete evidence for these claims.

\section{Confounding Factors}

The inconsistent findings in verbal memory functions of an individual with ASD may also be attributed to the following confounding factors.

Experimental Design: Task complexity would greatly affect the results obtained in different research studies. For example, simple tasks, such as digit span and letter or word recall, would not impair the performance of ASDs. ${ }^{58,66,67}$ These studies may not therefore be able to find impairment on verbal memory in individuals with ASD when compared with normal developing individuals. In addition, some studies have employed fewer verbal materials and they have also been restricted to simple common words moreover, these studies have also reported intact verbal performance in ASD individuals. ${ }^{49}$ However, these studies may have overestimated the verbal memory ability of ASDs.

Developmental Trajectories: The chronological age of the ASDs is another factor which contributes to the inconsistent findings on their verbal memory performance. Evidence has shown that the verbal memory impairment is more severe in the younger ASD population. Simple tasks, such as letter or number recall, have been reported to show impairment in ASDs with a mean age of $6.5^{46}$ whereas it has been reported as intact in teenage and adult samples. ${ }^{58,67}$ A study which included both adults with ASD and children/adolescents with ASD also reported that children/adolescents with ASD react more slowly when they give the correct responses in different tasks which examine verbal memory performance. ${ }^{67}$ It is suggested that this may be due to the plasticity of the developing brain in children/adolescents with ASD when they adjust to and accommodate their inborn memory deficits. Therefore, children/adolescents with ASD may have a profounder impairment in verbal memory functions than their adult counterparts.

Level of Functioning: Various studies have found that low-functioning ASD individuals Lo-AUT perform worse than high-functioning ASD individuals (Hi-AUT) in verbal memory performance. ${ }^{63}$ Most of the studies did not classify their ASDs into Hi-AUT and Lo-AUT, and therefore they blurred the real situation. There should be an association between the level of functioning and the verbal memory functions, i.e., the higher the intelligent quotient of the ASD, the better the verbal memory performance. In addition, two studies administered the same test; the group of children with ASD achieving a lower Verbal IQ showed impairment whereas the other one showed intact verbal memory performance ${ }^{58,61}$. Therefore, it is suggested that the level of functioning would also be a possible confounding factor in the inconsistent results on verbal memory performance of ASDs (Table 1).

Table I Selected research articles for review

\begin{tabular}{|c|c|c|c|c|c|c|}
\hline Reference & Groups & $\begin{array}{l}\text { Sample } \\
\text { Size }\end{array}$ & $\begin{array}{l}\text { Age } \\
\text { (Years) }\end{array}$ & Matched Criteria & $\begin{array}{l}\text { Measures of Verbal } \\
\text { Memory }\end{array}$ & $\begin{array}{l}\text { Impairments } \\
\text { found in } \\
\text { Autistic } \\
\text { Sample }\end{array}$ \\
\hline \multirow[t]{5}{*}{ Williams DL et al. ${ }^{67}$} & Normal (Adult) & 25 & 26.8 & Age, FSIQ,VIQ,\& PIQ & N - Back Letter Task & \\
\hline & Normal (Children) & 44 & 12.4 & & $\begin{array}{l}\text { Wechsler Memory } \\
\text { Scale }\end{array}$ & No \\
\hline & Hi-AUT (Adult) & 31 & 26.6 & & - Letter Number & \\
\hline & Hi-AUT (Children) & 24 & 11.8 & & WRAML & No \\
\hline & & & & & - Number/Letter & No \\
\hline \multirow[t]{2}{*}{ Bowler DM et al. ${ }^{69}$} & Normal & 21 & 16 & $\begin{array}{l}\text { Age,VIQ, Mother } \\
\text { Language }\end{array}$ & RAVLT & No \\
\hline & High-AUT & 21 & 19 & & & \\
\hline \multirow[t]{2}{*}{ Whitehouse AJO et al. ${ }^{70}$} & Normal & 20 & 8.3 & $\begin{array}{l}\text { Verbal Mental Age \& } \\
\text { Reading Ability }\end{array}$ & $\begin{array}{l}\text { Recall Task with Print } \\
\text { Word Stimuli }\end{array}$ & No \\
\hline & ASD & 20 & 10.9 & & & \\
\hline \multirow[t]{3}{*}{ Tyson $\mathrm{K}$ et al. ${ }^{71}$} & Normal & 34 & 13.9 & Age, Gender, \&N-VIQ & $\begin{array}{l}\text { CVLT-2nd Edition } \\
\text { II/\#CVLT-Children's } \\
\text { Version }\end{array}$ & Yes \\
\hline & Optimal-AUT & 34 & 12.8 & & & \\
\hline & High-AUT & 44 & 13.9 & & & \\
\hline
\end{tabular}


Table Continued...

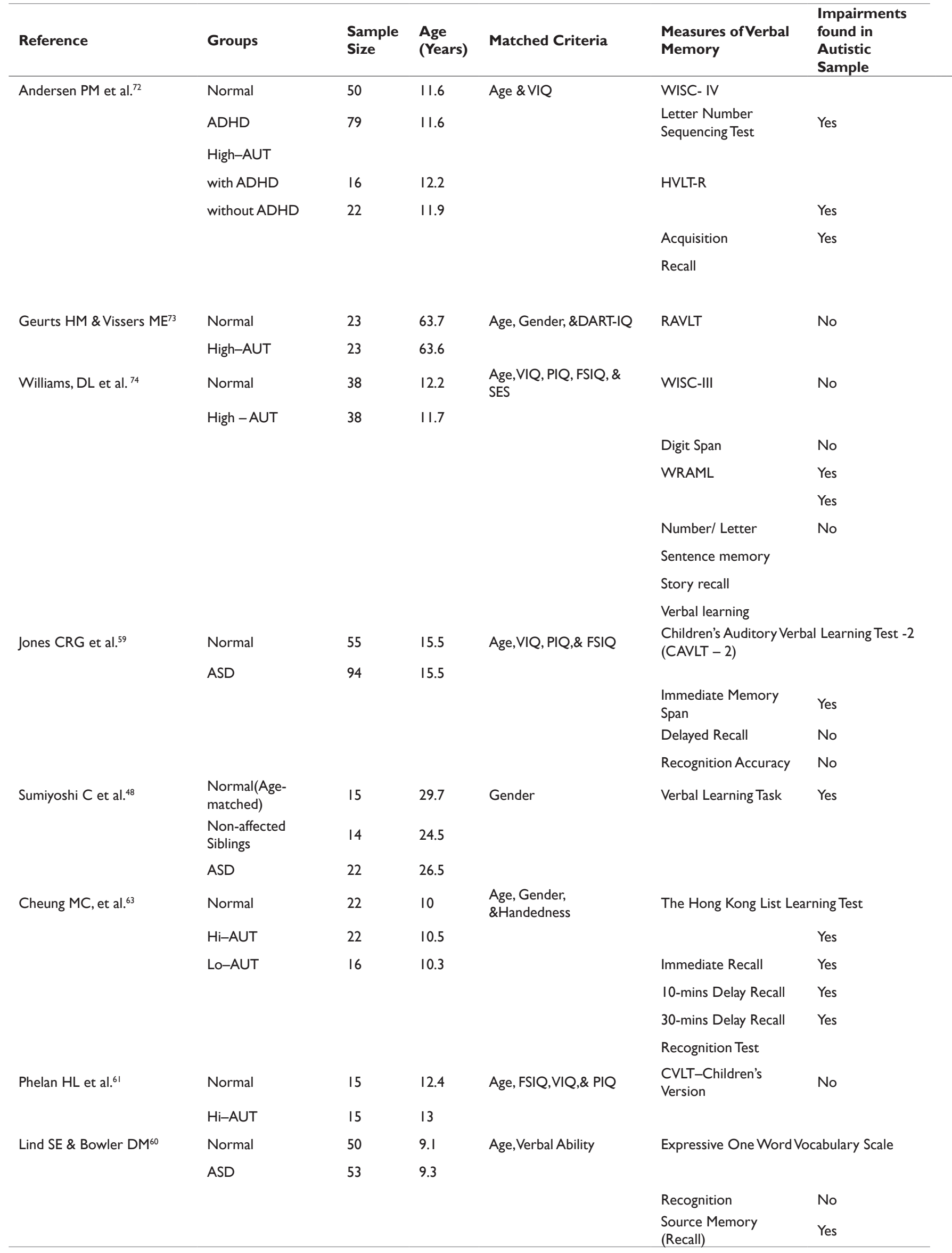


Table Continued..

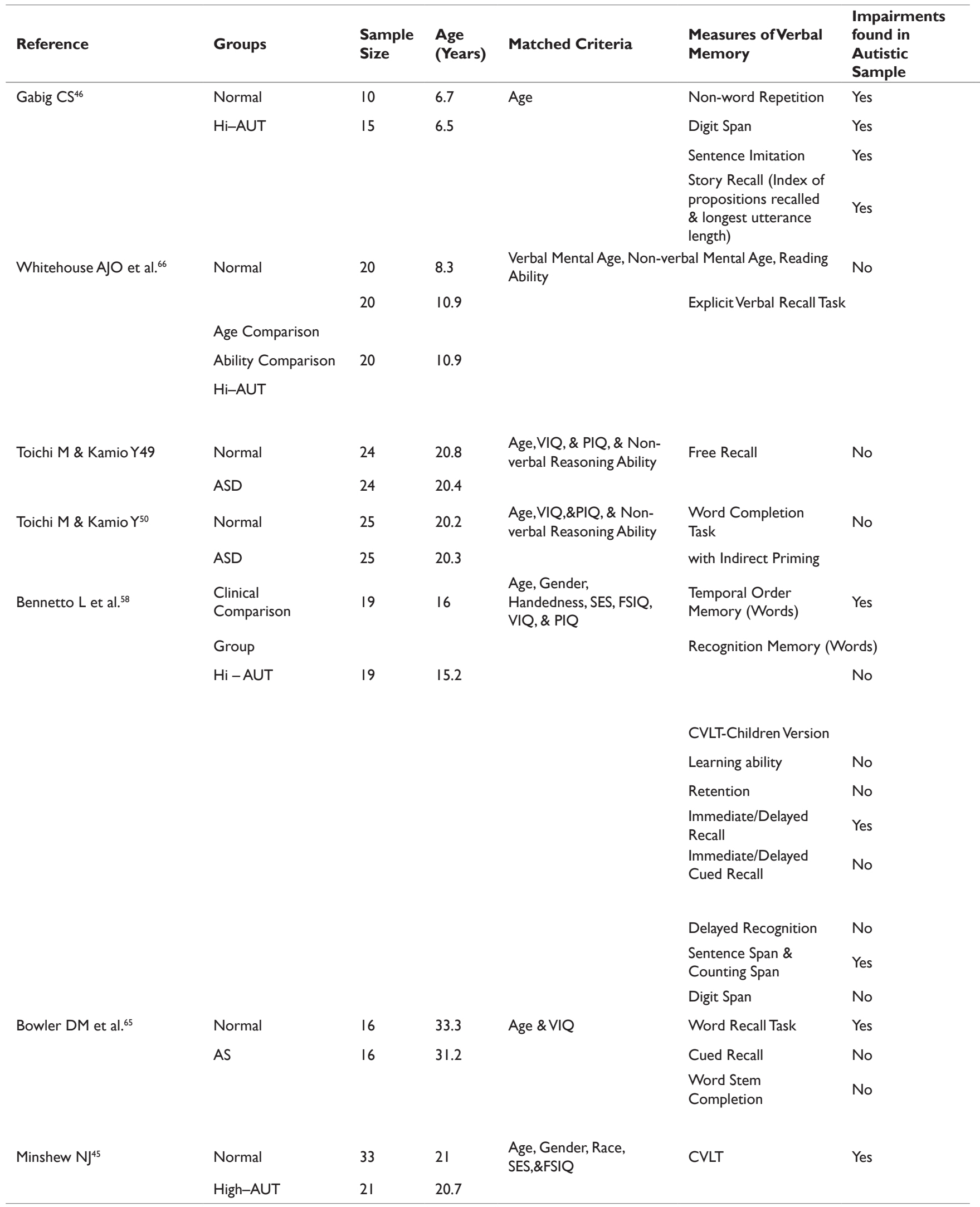

Note: \#for children with an age of 17 or below.

High-AUT: High-functioning Autism; Low-AUT: Low-functioning Autism; AS: Asperger Syndrome; ASD: Autism Spectrum Disorder; ADHD: Attention Deficit Hyperactive Disorder; DART-IQ: Dutch adult reading test IQ; VIQ: Verbal IQ; N-VIQ: Non-verbal IQ; PIQ: Performance IQ; FSIQ: Full scale IQ; SES: Socioeconomic status ; CVLT: California Verbal Learning Test; HVLT-R: Hopkins Verbal Learning Test - Revised; RAVLT: Rey Auditory Verbal Learning Test; WRAML: Wide Range Assessment of Memory and Learning;WISC:Wechsler Intelligence Scale for Children 


\section{Conclusion}

In the present article, we discovered that previous research studies have focused on Hi-AUT in which Lo-AUT individuals were rare. Several studies have combined Hi-AUT and Lo-AUT for analysis but there were no comparisons between them. Although some studies have preliminarily shown uneven memory abilities across the spectrum, ${ }^{58,61,63}$ there is still an urgent need to implement more studies on Lo-AUT and to compare their performance with $\mathrm{Hi}-$ AUT in order to have a more comprehensive understanding of the verbal memory profile across the spectrum. Ideally, studies including both neuropsychological assessments and brain measures would potentially be able to identify the underlying mechanism leading to the memory deficit in ASDs. Added to that, most of the studies presented used either teenage or adult samples; more studies need to be done on children to keep track of the developmental changes in ASDs. Longitudinal studies of memory functions in ASDs would shed light on the developmental factors that may contribute to the impaired verbal memory performance in ASD. Memory development in normal individuals follows a well-documented trajectory ${ }^{68}$ and ASD as a neuro-developmental disorder, it is suggested the delayed or abnormal memory development of ASDs in their early years may have cumulative consequences as they are growing up. More evidence is therefore required before a valid conclusion can be drawn. Finally, it has been suggested that ASD individuals are less efficient in initialing cognitive strategies to organize information. One study has reported an increase in performance from a recall of random word list to a categorized word list for ASDs. However, their performance nevertheless remained worse than that of normal developing individuals ${ }^{48}$ Further research with more vigorous experimental designs is required to support the claim, and we intend to devise interventions and measures to overcome the incapability of ASD individuals to initiate organization strategies. This will advance our theoretical understanding as well as yield practical value in enhancing the memory functioning of individuals with ASD and in improving their learning and academic performance.

\section{Acknowledgments}

None.

\section{Conflicts of interest}

Author declares there are no conflicts of interest.

\section{Funding}

None.

\section{References}

1. American Psychiatric Association. Diagnostic and statistical manual of mental disorder. (4th ed), Text Rev, Washington, USA. 2000.

2. Bailey A, Le Couteur A, Gottesman I, et al. Autism as a strongly genetic disorder: evidence from a British twin study. Psychological Medicine. 1995;25(1):63-77.

3. Newschaffer CJ, Fallin D, Lee NL. Heritable and nonheritable risk factors for Autism Spectrum Disorder. Epidemiol Rev. 2002;24(2):137-153.

4. Verhoeven JS, Cock PD, Lagae L, et al. Neuroimaging of autism. Neuroradiology. 2010;52(1):3-14.

5. Matson JL, Kozlowski AM. The increasing prevalence of autism spectrum disorder. Research in Autism Spectrum Disorders. 2011;5(1):418-425.

6. Newschaffer CJ, Croen LA, Daniels J, et al. The epidemiology of autism spectrum disorders. Annu Rev Public Health. 2007;28: 235-258.
7. Matson JL, Kozlowski AM. The increasing prevalence of autism spectrum disorder. Research in Autism Spectrum Disorders. 2011;5(1):418-425.

8. Wing L, Potter D. The epidemiology of autistic spectrum disorders; is the prevalence rising? Ment Retard Dev Disabil Res Rev 8(3): 151-161.

9. Kogan MD, Blumberg SJ, Schieve LA, Boyle CA, Perrin JM, et al. (2009) Prevalence of parent-reported diagnosis of Autism Spectrum Disorder among children in the US, 2007. Pediatrics. 2002;124(5):1395-1403.

10. Happe F. Autism: cognitive deficit or cognitive style? Trends Cogn Sci. 1999;3(6):216-222.

11. O'Connor N, Hermelin B. The memory structure of autistic idiot-savant mnemonists. Br J Psychol. 1989;80(pt 1):97-111.

12. Baron-Cohen S, Leslie AM, Frith U. Does the autistic child have a 'theory of mind'? Cognition. 1985;21(1):37-46.

13. Castelli F, Frith C, Happe F, et al. Autism, Asperger syndrome and brain mechanisms for the attribution of mental states to animated shapes. Brain. 2002;125(Pt 8):1839-1849.

14. Frith U, Frith CD. Development and neurophysiology of mentalizing. Philos Trans R Soc Lond B Biol Sci . 2003;358(1431):459-473.

15. Gallagher HL, Frith CD. Functional imaging of 'theory of mind'. Trends Cogn Sci. 2003;7(2):77-83.

16. Happe F, Ehlers S, Fletcher P, et al. 'Theory of mind' in the brain. Evidence from a PET scan study of Asperger syndrome. Neuroreport. 1996;8(1):197-201.

17. Mottron L, Peretz I, Menard E. Local and global processing of music in highfunctioning persons with autism: beyond central coherence? Journal of Child Psychology \& Psychiatry. 2000;41(8):1057-1065.

18. Plaisted K. Reduced generalization in autism: an alternative to weak central coherence. In The Development of Autism: Perspectives from Theory and Research. In: Burack JA et al. (Eds.), Erlbaum, USA. 2001. p.149-169,

19. Damasio AR, Maurer RG. Neurological model for childhood autism. Arch Neurol. 1978;35(12):777-786.

20. Hill EL. Executive dysfunction in autism. Trends Cogn Sci. 2004;8(1):26-32.

21. Pennington BF, Ozonoff S. Executive functions and developmental psychopathology. J Child Psychol Psychiatry. 1996;37(1):51-87.

22. Sergeant JA, Geurts H, Oosterlaan J. How specific is a deficit of executive functioning for attention-deficit/hyperactivity disorder? Behav Brain Res. 2002;130:3-28.

23. Miller EK. The prefrontal cortex: complex neural properties for complex behavior. Neuron. 1999;22(1):15-17.

24. Miller EK, Cohen JD. An integrative theory of prefrontal cortex function. Annu Rev Neurosci . 2001;24:167-202.

25. Stuss DT, Benson DF. Neuropsychological studies of the frontal lobes. Psychol Bull. 1984;95(1):3-28.

26. Moscovitch M, Winocur G. Frontal lobes, memory, and aging. Ann N Y Acad Sci. 1995;769:119-150.

27. Benson DF, Stuss DT, Naeser MA, et al. The long term effects of prefrontal leucotomy. an overview of neuropsychologic residuals. J Clin Neuropsychol. 1981;3(1):13-32.

28. Pigott S, Milner B. Memory for different aspects of complex visual scenes after unilateral temporal - or frontal - lobe resection. Neuropsychologia. 1993;31(1):1-15.

29. Fuster JM. Memory in the Cerebral Cortex. MIT Press, Cambridge, UK. 1995.

30. Goldman-Rakic PS. Anatomical and functional circuits in prefrontal cortex of nonhuman primates: Relevance to epilepsy. In: Jasper $\mathrm{HH}$ (Eds.), Epilepsy and the functional anatomy of the frontal lobe. Raven Press, New York, USA. 1995. p.51-62. 
31. Milner B, Petrides M, Smith ML. Frontal lobes and the tempora organization of memory. Hum Neurobiol. 1985;4(3):137-142.

32. Benson DF. Prefrontal abilities. Behav Neurol. 1993;6(2):75-81.

33. Fuster JM. Prefrontal cortex and the bridging of temporal gaps in the perception-action cycle. Ann N Y Acad Sci. 1990;608:318-329.

34. Petrides M. Impairments on non spatial self-ordered and externally ordered working memory tasks after lesions of the mid-dorsal part of the lateral frontal cortex in the monkey. J Neurosci. 1995;15(1 pt 1):359-375

35. Alexander MP, Stuss DT, Fansabedian N. California Verbal Learning Test: Performance by patients with focal frontal and non-frontal lesions. Brain. 2003;126(Pt6):1493-1503.

36. Gershberg FB, Shimamura AP. Impaired use of organizational strategies in free recall following frontal lobe damage. Neuropsychologia. 1995;33(10):1305-1333.

37. Stuss DT, Alexander MP. Does damage to the frontal lobes produce impairment in memory? Current Directions in Psychological Science. 2005;14(2):84-88.

38. Stuss DT, Alexander MP, Palumbo CL, et al. Organizational strategies of patients with unilateral or bilateral frontal lobe injury in word list learning tasks. Neuropsychology. 1994;8(3):355-373.

39. Grasby PM, Frith CD, Friston KJ, et al. Functional mapping of brain areas implicated in auditory -verbal memory function. Brain. 1993;116(Pt 1):1-20.

40. Fletcher PC, Frith CD, Grasby PM, et al. Brian systems for encoding and retrieval of auditory-verbal memory: An in vivo study in humans. Brain. 1995; 118(Pt 2):401-416.

41. Klingberg T, Roland PE. Right prefrontal activation during encoding, but not during retrieval, in a non-verbal paired-associates task. Cerebral Cortex. 1998;8(1):73-79.

42. Fletcher PC, Shallice T, Dolan RJ. The functional roles of prefrontal cortex in episodic memory: Encoding. Brain. 1998;121: 1239-1248.

43. Freedman M, Cermak LS. Semantic encoding deficits in frontal lobe disease and amnesia. Brain Cogn. 1986;5(1):108-114.

44. Kojima S, Kojima M, Goldman-Rakic PS. Operant behavioral analysis of memory loss in monkeys with prefrontal lesions. Brain Res. 1982;248(1):51-59.

45. Minshew N J, Goldstein G. Is autism an amnesic disorder? Evidence from the California Verbal Learning Test. Neuropsychology 1993;7(2):209-216.

46. Gabig CS. Verbal working memory and story retelling in school-age children with autism. Lang Speech Hear Serv Sch. 2008;39(4):498-511.

47. Minshew NJ, Goldstein G. The pattern of intact and impaired memory functions in autism. J Child Psychol Psychiatry. 2001;42(8):1095-1101.

48. Sumiyoshi C, Kawakubo Y, Suga M, et al. Impaired ability to organize information in individuals with autism spectrum disorders and their siblings. Neurosci Res. 2011;69(3):252-257.

49. Toichi M, Kamio Y. Verbal association for simple common words in high-functioning autism. J Autism Dev Disord. 2001;31(5):483-490.

50. Toichi M, Kamio . Long-term memory in high-functioning autism: Controversy on episodic memory in autism reconsidered. J Autism Dev Disord. 2003;33(2):151-161.

51. Delis DC, Kramer JH, Kaplan E, et al. California Verbal Learning Test. Psychological Corporation, New York, USA. 1987.

52. Chan AS, Kwok, I. Hong Kong List Learning Test: Manual and preliminary norm. Hong Kong: Department of Psychology, The Chinese University of Hong Kong, China. 1999.
53. Chan AS. Hong Kong List Learning Test (2nd ed.). Hong Kong: Department of Psychology and Clinical Psychology Centre, The Chinese University of Hong Kong, China. 2006.

54. Rey A. L'examenclinique en psychologie The clinical examination in psychology. PressesUniversitaires de France, Paris, France. 1964.

55. Taylor EM.Psychological appraisal of children with cerebral deficits. Harvard University Press, Cambridge, UK. 1959

56. Wechsler D. Wechsler Adult Intelligence Scale-Revised. The Psychological Corporation, New York, USA. 1981.

57. Wechsler D. Wechsler Memory Scale-Revised. The Psychological Corporation, San Antonio, Texas, USA. 1987.

58. Bennetto L, Pennington BF, Rogers SJ. Intact and impaired memory functions in autism. Child Development. 1996;67(4):1816-1835.

59. Jones CR, Happe F, Pickles A, et al. 'Everyday memory' impairments in autism spectrum disorders. J Autism Dev Disord. 2011;41(4):455-464.

60. Lind SE, Bowler DM. Recognition memory, self-other source memory, and theory-of-mind in children with autism spectrum disorder. J Autism Dev Disord. 2009;39(9):1231-1239.

61. Phelan HL, Filliter JH, Johnson SA. Brief report: memory performance on the California verbal learning test - children's version in Autism spectrum disorder. J Autism Dev Disord. 2011;41(4):518-523.

62. Rumsey JM, Hamburger SD. Neuropsychological findings in highfunctioning men with infantile autism, residual state. J Clin Exp Neuropsychol. 1988;10(2):201-221.

63. Cheung M-C, Chan AS, Sze SL, et al. Verbal memory deficits in relation to organization strategy in high- and low-functioning autistic children. Research in Autism Spectrum Disorder. 2010;4(4):764-771.

64. Alloway TP, Rajendran G, Archibald LMD. Working memory in children with developmental disorders. Journal of Learning Disabilities. 2009;42(4):372-382.

65. Bowler DM, Matthews NJ, Gardiner JM. Asperger's syndrome and memory: Similarity to autism but not amnesia. Neuropsychologia. 1997;35(1):65-70.

66. Whitehouse AJ, Maybery MT, Durkin K. Inner speech impairments in autism. J Child Psychol Psychiatry. 2006;47(8):857-865.

67. Cowan N. The development of memory in childhood. Psychology Press, Hove, England. 1998

68. Williams DL, Goldstein G, Carpenter PA, et al. Verbal and Spatial Working Memory in Autism. J Autism Dev Disord. 2005;35(6):747-755.

69. Bowler DM, Limoges E, Mottron L. Different verbal learning strategies in autism spectrum disorder: evidence from the Rey Auditory Verbal Learning Test. J Autism Dev Disord. 2009;39(6):910-915.

70. Whitehouse AJ, Maybery MT, Durkin K. Evidence against poor semantic encoding in individuals with autism. Autism. 2007;11(3):241-254.

71. Tyson K, Kelley E, Fein D, et al. Language and verbal memory in individuals with a history of Autism Spectrum Disorders who have achieved optimal outcomes. J Autism Dev Disord. 2014;44(3):648-663.

72. Andersen PN, Hovik KJ, Skogli EW, et al. Symptoms of ADHD in children with high-functioning Autism are related to impaired verbal working memory and verbal delayed recall. PLOS ONE. 2013;8(5):e64842.

73. Geurts HM, Vissers ME. Elderly with Autism: Executive functions and memory. J Autism Dev Disord. 2012;42(5):665-675.

74. Williams DL, Goldstein, G, Minshew NJ.The Profile of Memory Function in Children with Autism. Neuropsychology. 2006;20(1):21-29. 